\title{
Evaluation of Relative Permeability Tests One-Step with Bump Flow and Multi-Step
}

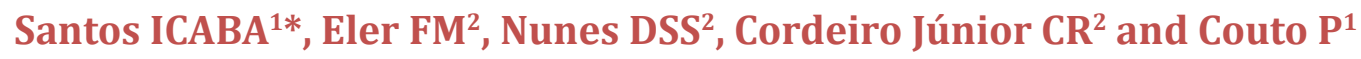 \\ ${ }^{1}$ Federal University of Rio de Janeiro, Civil Engineering Program, Brazil \\ ${ }^{2}$ COPPETEC, Rio de Janeiro, Brazil
}

\begin{abstract}
*Corresponding author: Isabelly Christinne ABA Santos, Federal University of Rio de Janeiro, Civil Engineering Program, Rio de Janeiro, RJ, Brazil, Tel: +5588988836855; Email: isabelly. santos@coc.ufrj.br
\end{abstract}

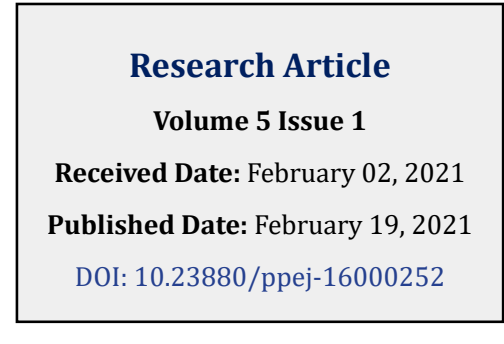

Research Article

Volume 5 Issue 1

Received Date: February 02, 2021

DOI: $10.23880 /$ ppej-16000252

\section{Abstract}

The relative permeability curves obtained in the laboratory are used in reservoir simulators to predict production and decide the best strategies for an oil field. Therefore, researchers are studying several procedures to obtain relative permeability curves, among them the multiple flow rates injection methods. Thus, this work proposes to develop an experimental procedure with multiple increasing flows (multi-step). To make this feasible, simulations were initially carried out at CYDAR, aiming to establish the flow rates and necessary the time to system stabilization, within the limits of the equipment. After that, the tests were carried out and the results obtained were the minimum time of 5 hours to stabilize the oil production and the differential pressure at each flow rate. The accounting and minimization of the capillary end effect in these tests were also evaluated. And the capillary pressure constraints contributed to minimize the number of possible solutions of the optimization problem improving the uniqueness of solution.

Keywords: Relative Permeability; Multiple Flows Rates; Cydar; Capillary End Effect

\section{Introduction}

Relative permeability is one of the most important data for reservoir engineering. The relative permeability curves obtained in the laboratory are used in reservoir simulators to predict oil production and decide the best strategies when exploring an oil field [1].

The tests to obtain these curves, in a water-oil system, are performed on rock samples from the reservoir or on representative outcrops. These are often subjected to displacement tests in an unsteady-state regime, in which an attempt is made to repeat the fluid displacement processes that take place in the oil reservoirs during secondary recovery activities. In the test, capillary and viscous forces act in the rock sample, as well as in the petroleum reservoirs
$[1,2]$.

Usually, this experiment is conducted by injecting water at a single constant flow rate. The experimental data obtained (differential pressure and produced volume of oil) is treated by analytical techniques, such as that of JBN [3], or by history matching, to obtain the parameterized relative permeability curves $\left(K_{\mathrm{r}}\right)$. However, these tests have limitations and conditions that are intrinsic to the laboratory, differently from what occurs in real reservoir flows, such as the capillary end effect. This happens due to a discontinuity in capillary pressure at the end sample outlet, causing an erroneous residual oil saturation $[4,5]$.

Besides, many mathematical methods used to infer the $K_{\mathrm{r}^{\prime}}$ as the JBN, do not consider the capillary forces, and 


\section{Petroleum \& Petrochemical Engineering Journal}

the method of history matching does not guarantee the uniqueness of solution if used in experiments with only one injection flow rate. This can generate bias in the relative permeability obtained in the laboratory [6].

In the exposed context, the present work proposes to develop an experimental procedure of multiple flow rates, to obtain the relative permeability curves, based on the theoretical methodology proposed by Lenormand \& Lenormand [7].

The greatest advantage of this method is the accounting of the oil that was retained in the sample due to the capillary end effect. Furthermore, there is the possibility of determining both the capillary pressure $\left(P_{c}\right)$ and relative permeability $\left(K_{\mathrm{r}}\right)$ in a single experiment and also to add a capillary pressure constraint to improve the uniqueness of the history match process.

\section{Relative Permeability Experiments}

During the drilling process of reservoirs, rock cores are extracted and undergo several laboratory tests to determine properties of relevance for oil extraction, such as porosity, permeability, and capillary pressure. Laboratory experiments must be carried out at reservoir conditions so that the results obtained are representative of the field.

In Figure 1, one of the most used core flooding experiments to determine the $\left(K_{\mathrm{r}}\right)$ is presented, it is called the unsteady state relative permeability. The Unsteady-State (USS) method consists of displacing the fluid that saturates the porous medium by injecting another fluid at constant flow or constant pressure [8].

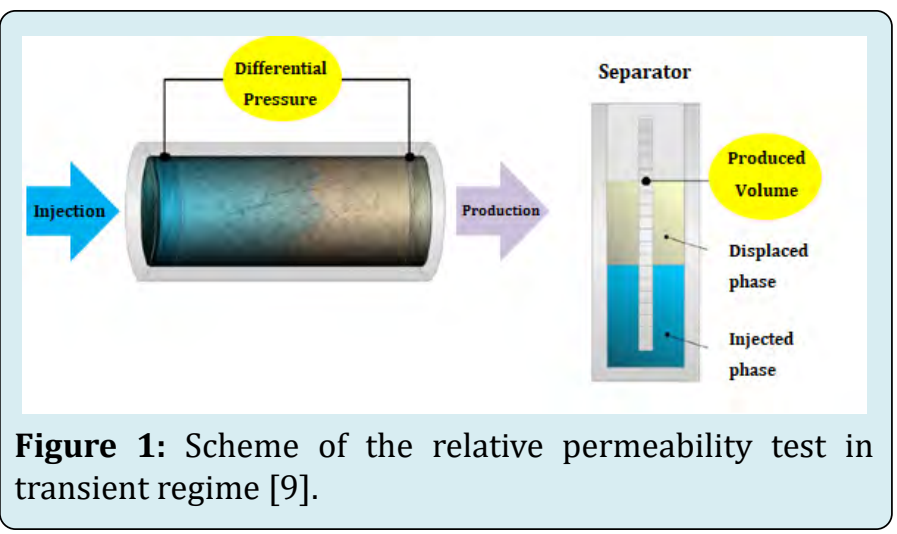

For a water-oil system, an oil-saturated core with immobile water $\left(S_{w i}\right)$ is first accommodated in the holder. This condition is representative of the initial state of the reservoir before the injection of fluids for its exploitation. Then, it is performed a water injection in the inlet face, while oil is produced at the outlet face. During the test, the produced oil volume and the differential pressure across the core are recorded.

The most used laboratory procedures in the water-oil relative permeability tests consists of the injection of water at a single flow rate, obtaining the residual oil saturation (Sor). The $S_{o r}$ can be greatly affected by the capillary end effect [10]. Thus, to minimize laboratory artifacts, a sudden increase in flow rate (bump flow) is performed at the end of the test. There is often an additional production of oil-related to the increase in differential pressure caused by the bump flow [1]. For identification purposes, the residual oil saturation $\left(S_{o r}\right)$ will be called remnant $S_{o r}\left(S_{o r}^{r e m}\right)$ when it is obtained in a test with only one injection rate and bump $S_{\text {or }}\left(S_{o r}^{\text {bump }}\right)$ when there is an increase in flow rate (bump flow).

\section{Converting Laboratory Data to Relative Permeability}

The relative permeability curves are inferred from the differential pressure and oil production data collected in these tests. The solution techniques normally applied are analytical solutions, such as JBN $[3,11]$, based on the BuckleyLeverett model for the displacement of immiscible fluids.

This method assumes simplifications such as onedimensional flow, negligible capillary pressure, and homogeneous porous medium. These simplifications hardly represent the reality of the displacement tests performed in the laboratory $[9,12]$.

Another technique very used is the history matching [13], which is a non-linear regression problem. The procedures of this nature use parameterized $K_{r}$ curves in a numerical flow simulator and an optimization process finds the parameters that best represent the experimental data. In this type of technique, the flow model can be simplified or not.

The advantage of this method is the possibility of including all effects that may be relevant in the actual displacement process, such as capillary pressure, medium heterogeneity, fluid compressibility, or gravity $[9,14]$. However, many software that uses the non-linear regression method do not include these effects in the theoretical model, analyzing the data up to $\left(S_{o r}^{r e m}\right)$, as in JBN [1].

\section{Multistep Procedure}

The multiple flow rates test should be considered over the traditional one (single flow rate plus bump flow), even if that lasts longer than this. Because the experiments in several increasing flow rates provide $K_{r}$ and $P_{c}$ curves simultaneously [10]. 


\section{Petroleum \& Petrochemical Engineering Journal}

In the technique proposed by Lenormand \& Lenormand [7], the test is started by injecting water into the sample at constant flow until the oil production and differential pressure stabilize. Then, the flow rate is increased until the new stabilization is achieved. This process is repeated using 5 to 10 steps of injection rates. With each rate, additional oil is produced and the average water saturation increases. With these data, it is possible to account for the capillary end effect and the amount of oil that was retained due to this phenomenon. At the end of the test, $\left(S_{o r}^{r e m}\right)$ is expected to be closer to the real $S_{o r}[4]$.

\section{Materials and Methods}

\section{Materials}

The rock samples used were homogeneous outcrops of the Berea Buff formation, obtained from Kocurek Industries. To minimize the influence of the type of sample in the tests, the same $20 \mathrm{~cm}$ core was split into smaller cores, approximately $5 \mathrm{~cm}$ each. The basic properties of these samples are shown in Table 1.

\begin{tabular}{|c|c|c|c|c|c|c|c|c|}
\hline Sample & $\begin{array}{l}\text { Length } \\
\text { L (cm) }\end{array}$ & $\begin{array}{c}\text { Diameter D } \\
(\mathbf{c m})\end{array}$ & $\begin{array}{c}\text { Absolute } \\
\text { Permeability } \\
K_{a b s} \text { (mD) } \\
\end{array}$ & $\begin{array}{c}\text { Porosity } \phi \\
\text { (frac.) }\end{array}$ & $\begin{array}{c}\text { Porous } \\
\text { Volume } V_{p} \\
\left(\mathrm{~cm}^{3}\right)\end{array}$ & \begin{tabular}{|c|} 
Irreducible \\
Water Saturation \\
$\left(\mathbf{S}_{\mathrm{wi}}\right)$ \\
\end{tabular} & $K_{r}$ test & $\begin{array}{c}\text { Alteration of } \\
\text { rock wettability }\end{array}$ \\
\hline Sample 1 & 4.78 & 3.82 & 597 & 0.225 & 11.99 & 0.202 & Bump flow & - \\
\hline Sample 2 & 4.84 & 3.82 & 602 & 0.227 & 12.24 & 0.187 & Multi-step & - \\
\hline Sample 3 & 4.9 & 3.82 & 601 & 0.228 & 12.5 & 0.208 & Multi-step & - \\
\hline Sample $3^{*}$ & 4.89 & 3.81 & 515 & 0.227 & 12.41 & 0.122 & Multi-step & $\begin{array}{c}\text { Saturation of rock } \\
\text { with SAN** }\end{array}$ \\
\hline Sample 4 & 4.61 & 3.82 & 597 & 0.228 & 11.79 & 0.169 & Multi-step & $\begin{array}{l}\text { Sample aging } \\
\text { with OMN*** }\end{array}$ \\
\hline
\end{tabular}

Table 1: Properties of the sample and fluids used in the USS experiment multiple flows.

*Sample subjected to a new $K_{r}$ test after cleaning; ${ }^{* *}$ SAN - naphthenic aqueous solution; ${ }^{* * *}$ Naphthenic Mineral Oil

The fluid used as the oil phase was the EMCA PLUS 70 and Naphthenic Mineral Oil, as the aqueous phase a brine with a composition equivalent to that of the Brazilian Pre-
Salt fields was used. The physico-chemical properties of fluids under test conditions are shown in Table 2.

\begin{tabular}{|c|c|c|}
\hline Fluid & Density $\rho \mathbf{( g / \mathbf { c m } ^ { \mathbf { 3 } } )}$ & Viscosity $\mu$ (cP) \\
\hline Mineral Oil (OM) & 0.856 & 23 \\
\hline Naphthenic Mineral Oil (OMN) & 0.848 & 23.5 \\
\hline Brine & 1.142 & 1.48 \\
\hline
\end{tabular}

Table 2: Physico-chemical properties of fluids at $21^{\circ} \mathrm{C}$ and ambient pressure.

\section{Methods}

Two types of coreflooding experiments were carried out: without changing the wettability of the rocks and with changing the wettability of the rocks (Table 1 ).

In general, in both testes, the USS water-oil relative permeability experiment at laboratory scale (Figure 2) followed the sequence of procedures proposed by Viegas [12]:

1. Cleaning the sample in Soxhlet apparatus, using methanol and methylbenzene (toluene).

2. The routine core analysis to obtain the basic properties of the samples, such as absolute permeability and effective porosity.

3. Total saturation of the samples with the brine of composition equivalent to the water of formation of the fields of the Brazilian Pre-Salt.

4. Centrifugation of samples in an oil environment to obtain irreducible water saturation $\left(S_{w i}\right)$.

5. Accommodation of the core sample on a hydrostatic confinement cell, using 1,000 psi of confining pressure.

6. Measurement of effective oil permeability at irreducible water saturation $\left(K_{r o} @ S_{w i}\right)$

7. Displacement of mineral oil by injection of brine to the state of residual oil saturation $\left(S_{o r}\right)$, with monitoring of 


\section{Petroleum \& Petrochemical Engineering Journal}

oil production $\left(N_{p}\right)$ and differential pressure $(\Delta P)$ :

7.1. With a constant flow of $1 \mathrm{~cm}^{3} / \mathrm{min}$ plus a bump flow of $4.0 \mathrm{~cm}^{3} / \mathrm{min}$ in the traditional test.

7.2. With five flow levels in the multistep test: 1.0, 2.0, $4.0,8.0$ and $10 \mathrm{~cm}^{3} / \mathrm{min}$.

7.3. Measurement of effective water permeability in $S_{o r}$;

The tests on rock samples with alteration of wettability distinguished from the others due to the addition of the following procedures:

1. Sample 3*: Alteration of wettability with saturation of naphthenic aqueous solution (SAN): this procedure involved saturating the rock sample with the naphthenic aqueous solution for $24 \mathrm{~h}$ at 2,000 psi. The sample was removed from the saturation system and placed in the oven for the drying step. Subsequently, the sample was subjected to new saturation with balanced brine of a composition equivalent to that of the Brazilian presalt for $24 \mathrm{~h}$ at 2,000 psi. Subsequently, the sample was sent to determine the $S_{w i}$ in the centrifuge, and was subsequently confined to the coreholder without carrying out the aging stage.

2. Sample $4^{*}$ : Alteration of wettability with naphthenic mineral oil (OMN): this procedure involved the injection of a naphthenic organic solution diluted in mineral oil in the rock sample at $S_{w i}$ About 8 injections with OMN were performed on alternate days, with a flow rate of $0.5 \mathrm{~cm}^{3} /$ min and a flow rate of $1.0 \mathrm{~cm}^{3} / \mathrm{min}$ until stabilization $K_{r o}$ in the $88 \%$ range (aging stage).

The experimental data was used to estimate the relative permeability and capillary pressure by history matching. The software CYDAR ${ }^{\circledR}$ was used to estimate the parameters of the used models: LET model [15] for relative permeability and $\log \left(S^{\beta}\right)$ model [16] for capillary pressure.

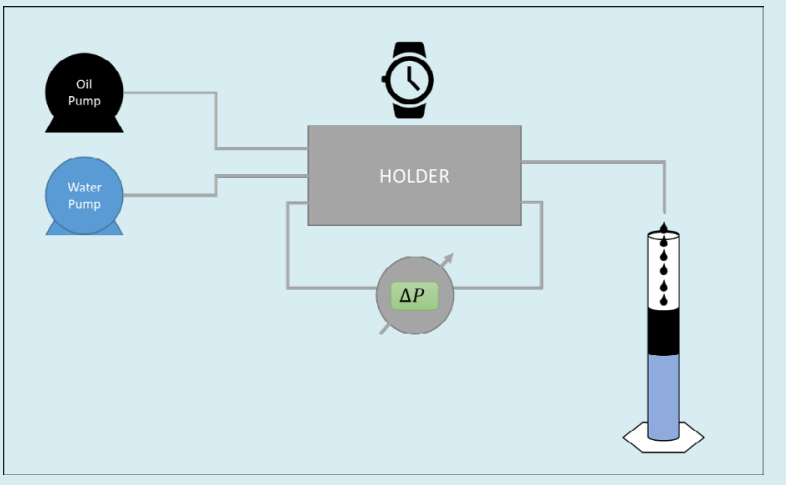

Figure 2: Schematic diagram of the relative permeability equipment under laboratory conditions.

\section{Results and Discussion}

\section{Analysis of Water-0il Relative Permeability Tests Considering Capillary Pressure}

Figure 3 shows the experimental data from Sample 1, in which water was injected at a flow rate of $1.0 \mathrm{~cm}^{3} / \mathrm{min}$ for 23 hours. At the end of this first injection, $6.25 \mathrm{~cm}^{3}$ of oil was produced. The bump flow, with a flow rate of $4 \mathrm{~cm}^{3} / \mathrm{min}$, was carried out afterward and the $\Delta P$ stabilized after 1 hour and there was no additional oil production. Returning to a flow rate of $1.0 \mathrm{~cm}^{3} / \mathrm{min}$, the stabilized after $30 \mathrm{~min}$. Thus, the traditional test with the bump flow was completed in $24.5 \mathrm{~h}$

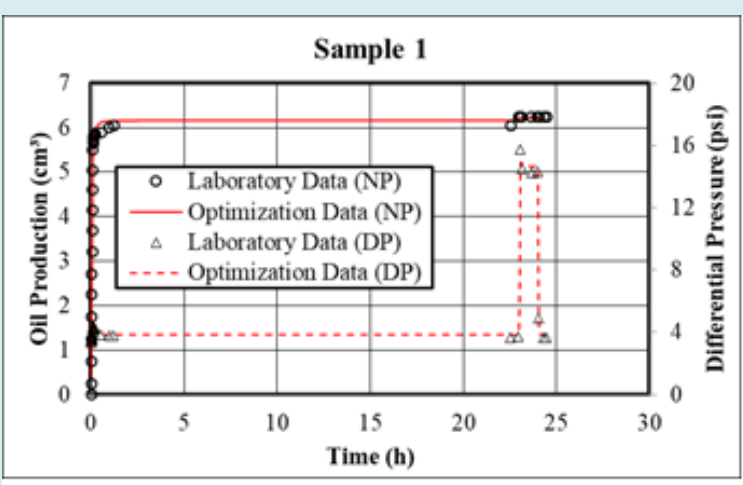

Figure 3: Experimental data obtained in the traditional water-oil relative permeability test with bump flow (points) and data obtained by the history-matching process (solid line) to obtain $\mathrm{Kr}$ and Pc curves. a) Differential pressure versus time. b) Volume of produced oil versus time.

Figure 4a shows the capillary pressure curve estimated for the imbibition test. As shown by Abdallah et, al. [17] the sample showed results from a water-wet sample, where the capillary pressure remains positive during most of the saturation range, as shown in Figure 4a Thus, water saturation preferably increases first in the smallest pores, due to wetting forces, so the displacement occurs from the smallest to the largest pores, and the water increasingly occupies the throat of pores that were previously filled with oil, as shown in Figure 4 b, which presents the relative permeability.

According to Masalmeh [18], water-wet samples have very well-defined residual oil saturation and are insensitive to the flow rate, that is, $S_{\text {or }}$ is not affected by the increased flow rate. Analyzing Figures 3 and 4, it is possible to infer that the sample is water-wet, so there was no additional production associated with bump flow, so $S_{o r}^{r e m}$ was equal to $S_{o r}^{\text {bump }}$ (Figure 3b). 


\section{Petroleum \& Petrochemical Engineering Journal}

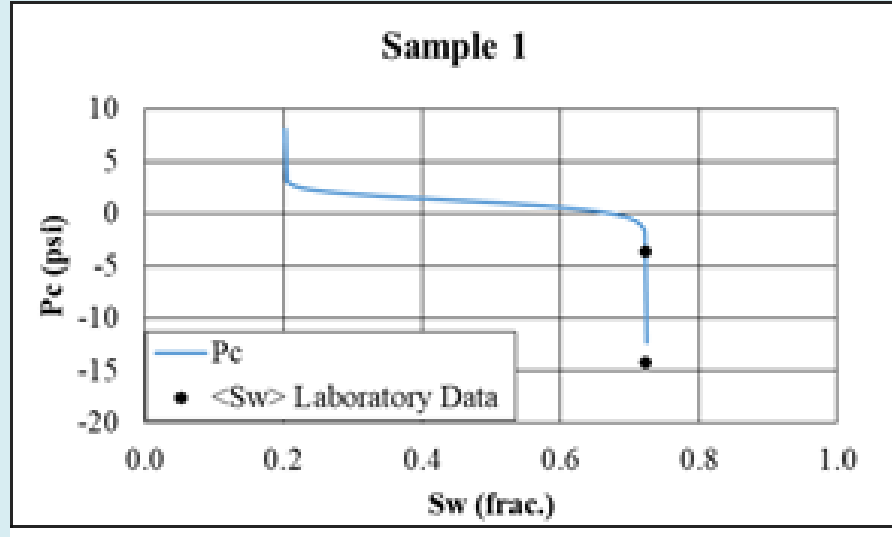

(a)

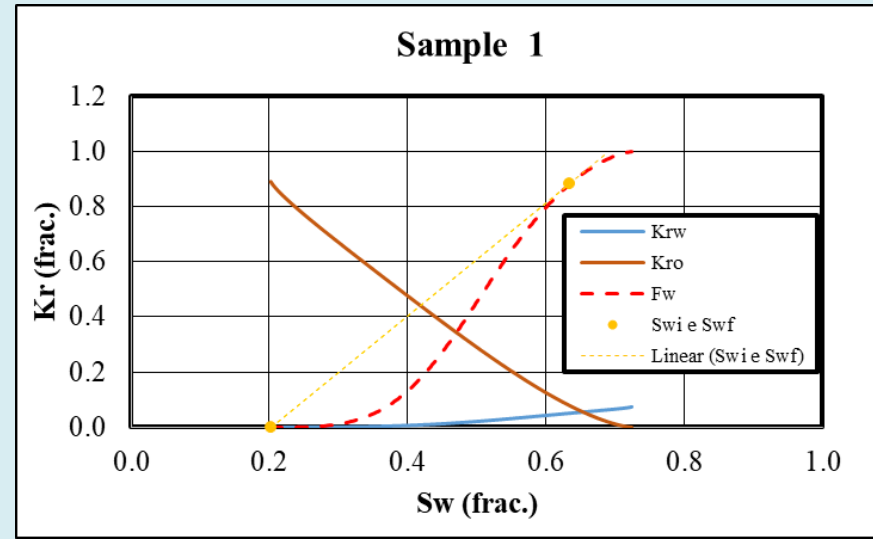

(b)

Figure 4: Curves estimated in CYDAR, using history matching. a) Capillary pressure curve as a function of water saturation. b) Relative permeability curves as a function of water saturation.

In Figure 5, the data of Sample 2 and Sample 3 are presented, which were submitted to the multistep process. In Figure $5 \mathrm{a}$ it is identified that in the first test flow the Sample 1, $7.80 \mathrm{~cm}^{3}$ of oil was produced, and at the end of the 30 hours test, $7.93 \mathrm{~cm}^{3}$ had been produced, that is, even increasing the flow 10 times, there was a gain of only $1.7 \%$ in the volume of fluid produced about a flow of $1.0 \mathrm{~cm}^{3} /$ min. While in Sample 3, shown in Figure 5b, the first flow of $1.0 \mathrm{~cm}^{3} /$ min produced $7.25 \mathrm{~cm}^{3}$ during the initial 5 hours, ending the test with $7.50 \mathrm{~cm}^{3}$ of displaced oil. Thus, the $10-$ fold increase in flow provided a small gain of $3.4 \%$ of the oil produced about the initial flow.

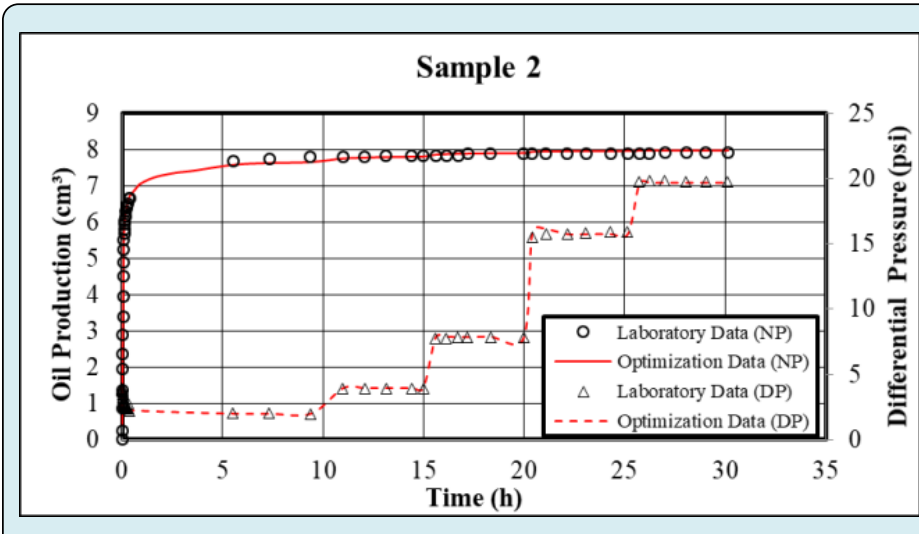

(a)

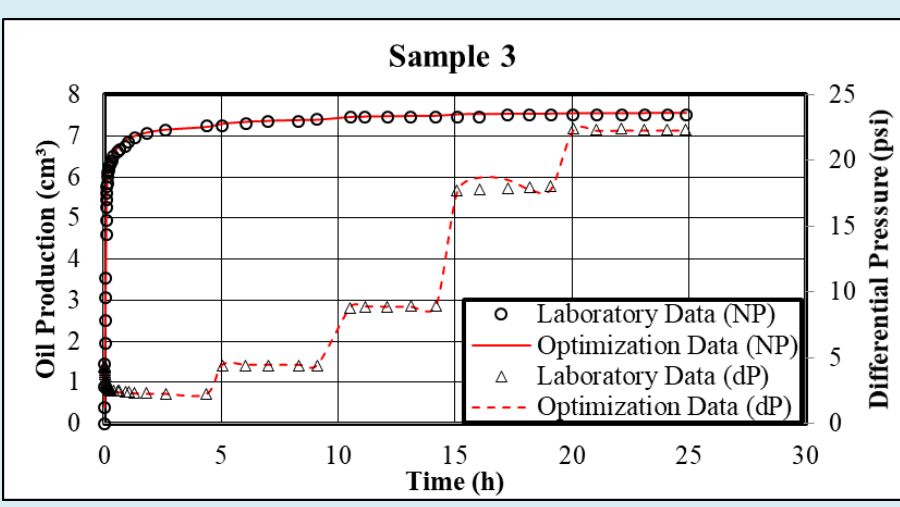

(b)

Figure 5: Experimental data obtained in the multi-step water-oil relative permeability test (points) and data obtained by the history-matching process (solid line) to obtain $\mathrm{K}_{\mathrm{r}}$ and $\mathrm{P}_{\mathrm{c}}$ curves. a) Sample 2. b) Sample 3.

The capillary pressure and relative permeability curves, presented in Figure 6 and Figure 7, fitted the experimental data with precision, as shown in Figure 5. In Figure 6, the $P_{c}$ curves are positive in most of the water saturation range, representing water-wet samples. Also, adapting the calculation of the USBM index for the imbibition test, which measures the wettability using the areas under the positive and negative capillary pressure curves, this water wettability is confirmed, since the results are values greater than 1 [17]. 


\section{Petroleum \& Petrochemical Engineering Journal}

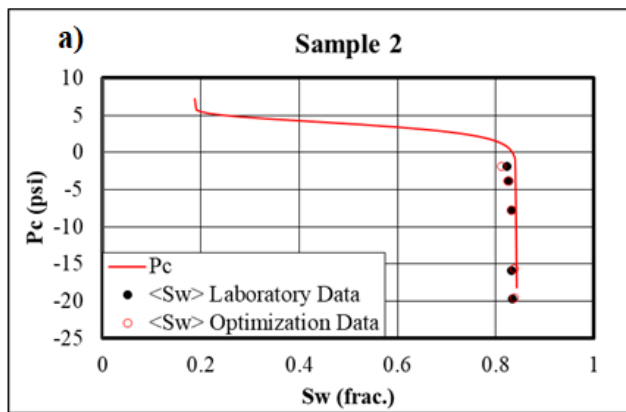

(a)

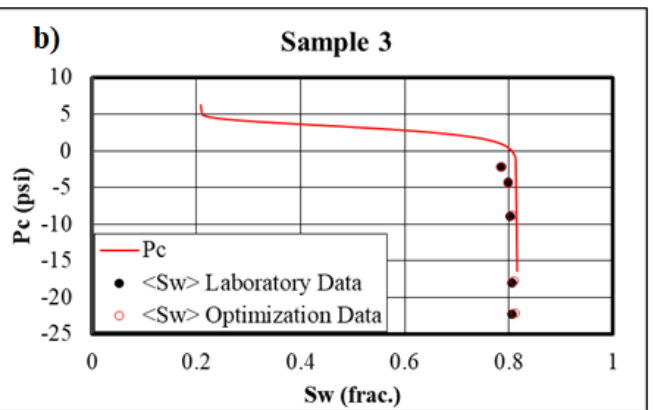

(b)

Figure 6: Capillary pressure estimated by history matching versus experimental average water saturation and optimized average water saturation. a) Sample 2. b) Sample 3.

Besides, interpreting the $K_{r}$ curves in Figure 7, it is noticed that water begins to enter the smaller pores first, and as the water saturation increases, the pores are filled sequentially from the smallest to the largest, as well as a preferably wettable sample. water, as explained by Crotti [19].

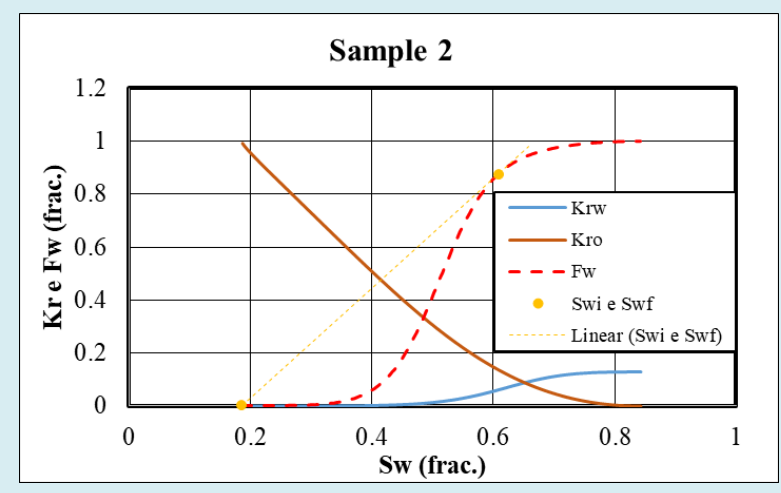

(a)

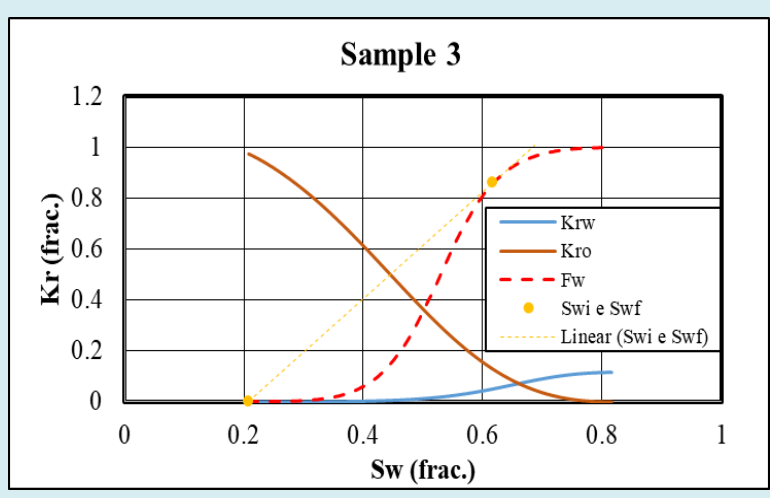

(b)

Figure 7: Relative permeability estimated by history matching as a function of water saturation. a) Sample 2. b) Sample 3.

The average water saturation $\left\langle S_{w}\right\rangle$ versus capillary pressure data presented in Figure 6 was obtained by the procedure proposed by Lenormand \& Lenormand [7]. This procedure assumes that at the end of each flow rate, as oil production stops, the oil phase is not moving, therefore the differential pressure for this phase equals to zero $\left(\mathrm{P}_{0}\right)$. This implies that the differential pressure measured across the sample is the water phase differential pressure $\left(\mathrm{P}_{w}\right)$ (Figure 8 and Equation 1). In Equations 2 and 3 it is demonstrated how these calculations are performed.

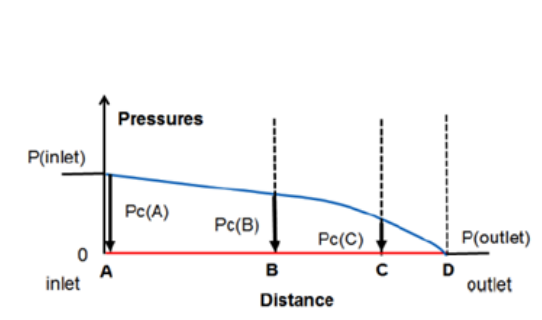

a)

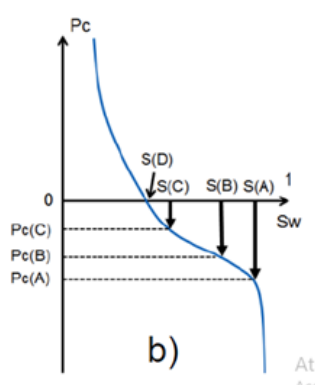

b)

Figure 8: USS displacement. a) Pressure profile at equilibrium (water in blue and oil in red). The arrows represent the capillary pressure in different positions across the sample length. b) Determination of the correspondent water saturation in the capillary pressure curve [7]. 


\section{Petroleum \& Petrochemical Engineering Journal}

$$
\begin{gathered}
P_{w}=\Delta P(1) \\
P_{C}=P_{O}-P_{w}=0-\Delta P \\
P_{c}=-\Delta P
\end{gathered}
$$

This data points of $P_{\mathcal{C}}$ vs $\left\langle S_{w}\right\rangle$ were used in CYDAR as an experimental constraint to the estimated capillary pressure, improving the solution uniqueness problem, faced in history matching process. Comparing the experimental and estimated data it is possible to conclude that the constraint was satisfied with great precision.

\section{Tests with Change in Wettability \\ Using Naphthenic Mineral Oil in Aging}

Sample 4 was submitted to the aging process, aiming to change its wettability, since sample 2 and 3 taken from the same core were wettable to water.

Figure 9 shows the experimental data obtained in sample 4 , submitted to the multiple flow test with aging using $0 M N$. The test started with the injection of water at a flow rate of $1 \mathrm{~cm}^{3} /$ min, stabilizing $N_{p}$ and DP in $5 \mathrm{~h}$ after starting the pump. The volume obtained, in the inverted separator, in this first flow was $6.85 \mathrm{~cm}^{3}$. At the end of the test, $7.75 \mathrm{~cm}^{3}$ of the produced fluid was displaced. Thus, the increase of 10 times in the flow, provided a gain of approximately $13 \%$ of oil produced in relation to the initial flow, that is, there was a significant change in the $S_{o r}^{r e m}$. During the test stabilization at $1 \mathrm{~cm}^{3} / \mathrm{min}$, the sample's $S_{o r}^{r e m}$ was 0.25 , while at the end of the test, at $10 \mathrm{~cm}^{3} / \mathrm{min}$, there was a decrease in oil saturation to 0.17 .

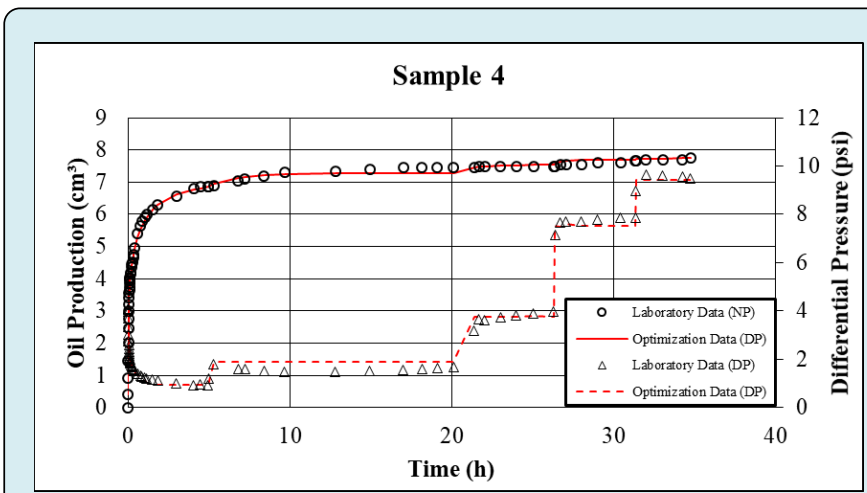

Figure 9: Experimental data obtained in the wateroil relative permeability tests of multistep (points and triangles) and the adjusted curves (solid line) from the estimated $\mathrm{Kr}$ and $P_{C}$ curves.

Additionally in Figure 9, it is analyzed that the estimated water-oil relative permeability and capillary pressure curves, shown below in Figure 10a and Figure 10b, respectively, adjusted the data well.

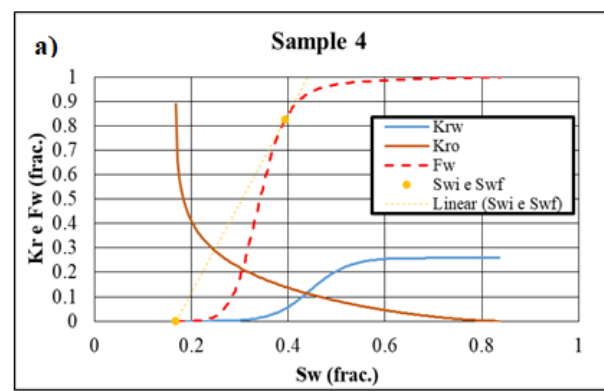

(a)

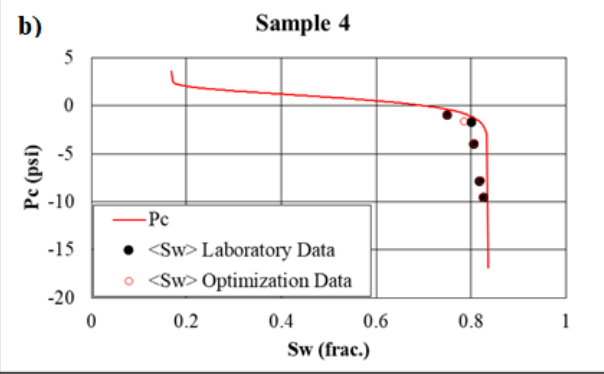

(b)

Figure 10: Curves estimated in CYDAR, using history matching. a) Relative permeability curves as a function of water saturation. b) Capillary pressure curve as a function of water saturation.

Interpreting the $K_{r}$ curves in Figure 10a, the crossing of the curves occurs at $S_{w}<0.5$, common for oil-wettable samples, that is, water begins to enter the larger capillary tubes, and as the water saturation increases, the tubes are filled sequentially from largest to smallest [19]. However, analyzing the $P_{c}$ curve, Figure 10b, the capillary pressure remains positive during most of the saturation range, crossing the axis at approximately 0.7 saturation, and the USBM index was 0.658 . Thus, it was not possible to conclude whether the sample is strongly wettable to water or oil, being considered with mixed wettability. Another point to be noted in Figure 10b, was how much the experimental points of $\left\langle S_{w}\right\rangle$, calculated from Equation 3, approached the water saturation on the face, estimated on the $P_{\mathcal{C}}$ curve.

\section{Using naphthenic aqueous solution in saturation}

Sample 3 was subjected to a new test of relative permeability water-oil of multiple flows, after cleaning, being named sample $3 *$. In this test the sample was saturated $100 \%$ in naphthenic aqueous solution, aiming to change its 
wettability.

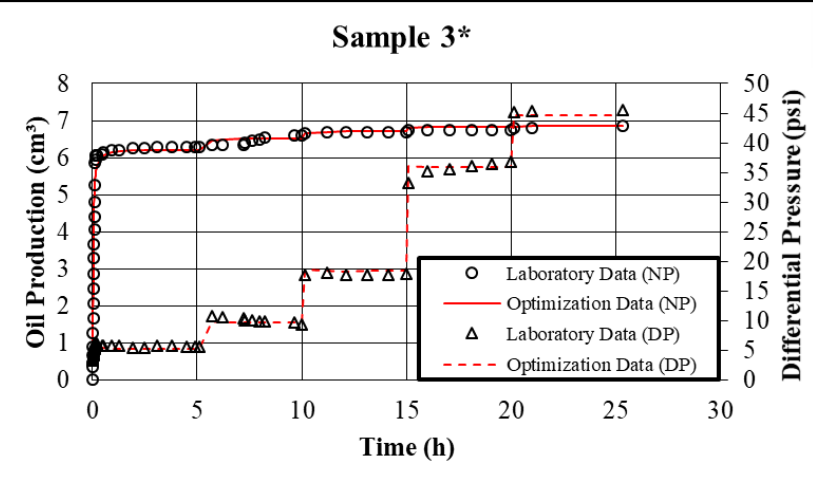

Figure 11: Experimental data obtained in the wateroil relative permeability tests of multistep (points and triangles) and the adjusted curves (solid line) from the estimated $\mathrm{Kr}$ and $P_{c}$ curves.
The experimental data obtained in sample $3^{*}$ are shown in Figure 11. The volume obtained, in the stabilization of the first flow, $1 \mathrm{~cm}^{3} / \min$, was $6.30 \mathrm{~cm}^{3}$. At the end of the test, $6.85 \mathrm{~cm}^{3}$ of the produced fluid was displaced. Thus, the 10 -fold increase in flow, provided an increase of $6.6 \%$ of oil produced in relation to the initial flow.

Additionally in Figure 11, it is analyzed that the estimated water-oil relative permeability and capillary pressure curves, shown below in Figure 12a and Figure 12b, respectively, adjusted the data well.

The water-oil relative permeability curves, shown in Figure $12 \mathrm{a}$, intersect approximately at the $0.56 S_{w^{\prime}}$ showing a tendency of the sample to remain water-wettable. The capillary pressure curve has a USBM index of 0.44 , the crossing of the curve occurs approximately at 0.53 saturation (Figure 12b). Thus, it is not possible to conclude whether the sample is strongly wettable to water or oil, being considered with mixed wettability.

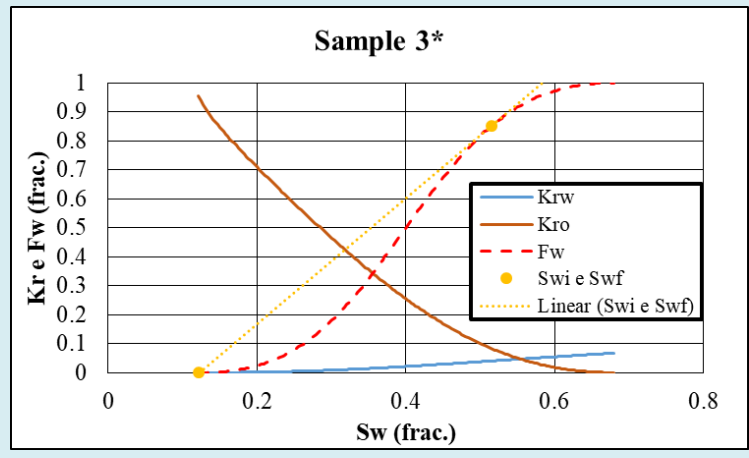

(a)

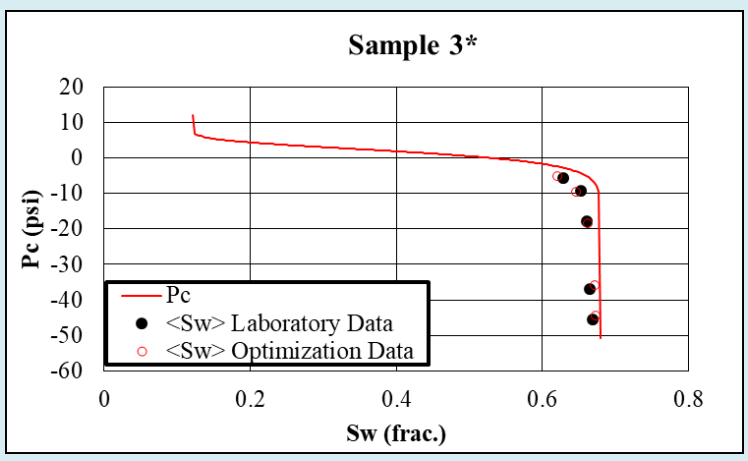

(b)

Figure 12: Curves estimated in CYDAR, using history matching. a) Relative permeability curves as a function of water saturation. b) Capillary pressure curve as a function of water saturation.

\section{Analysis of Saturation Profiles}

Saturation profiles at the end of each flow rate were also obtained by the history-matching process (Figure 13). It is possible to notice that the increase in flow rates causes an increase in the average water saturation and, consequently, an increase in oil production, as it was shown.

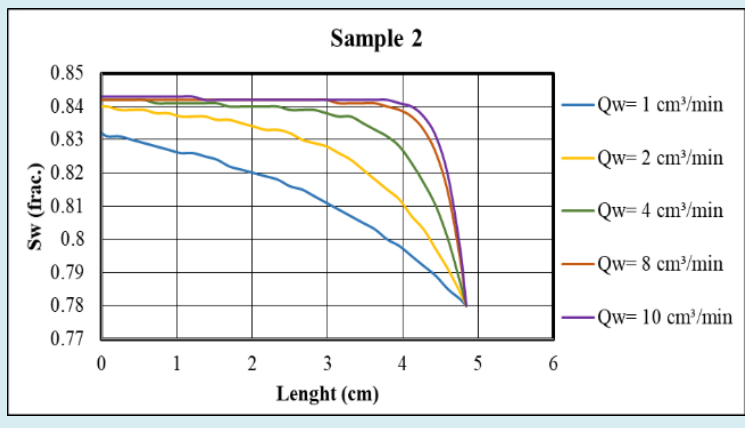

(a)

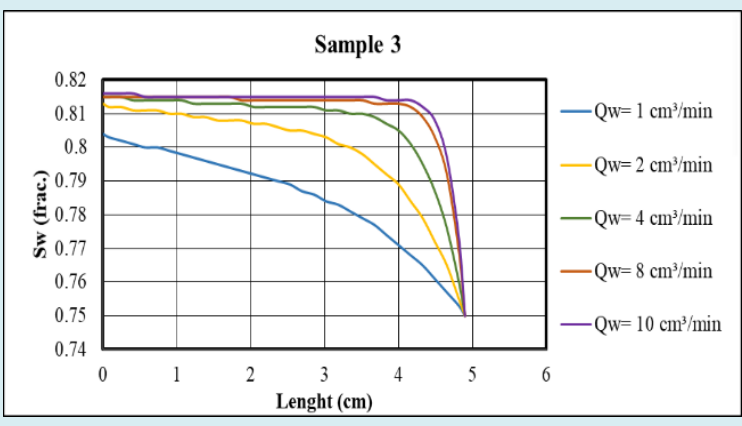

(b) 


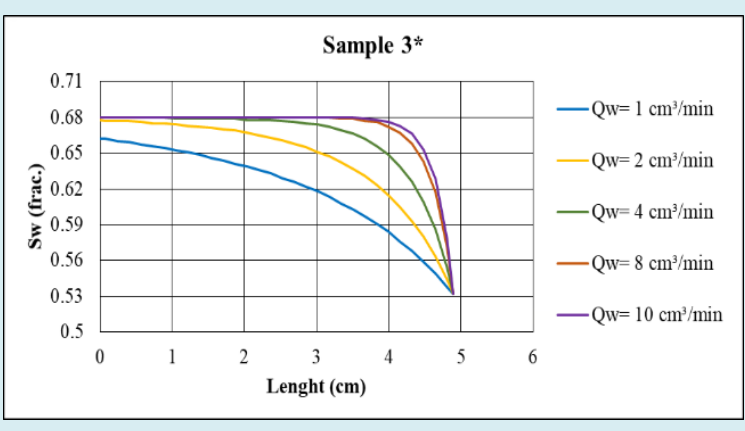

(c)

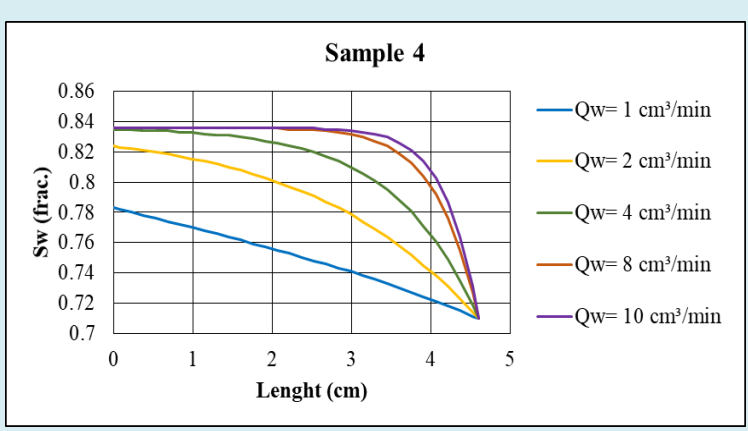

(d)

Figure 13: Saturation profile at the end of each flow, obtained by simulation in Cydar. a) Sample 1. b) Sample 2. c) Sample 3. d) Sample 4 .

In addition, the saturation profiles become more uniform over the length of the sample as the flow increases, converging on the sample's outlet face. In Figure 13, it is possible to observe that the oil is stuck at the end of the core sample, due to the edge effect [20].

\section{Conclusions}

From the relative permeability experiments carried out, in three sandstone samples of the same petrophysical properties, but comparing different methodologies-Sample 1 with conventional test; Samples 2, 3 and 4 with multi-step - it is possible to conclude that:

1. In high permeability water-wet and mixed-wet samples the stabilization of the differential pressure and produced volume is achieved in 5 hours, at each imposed flow rate.

2. In general, the samples submitted to the multi-step test further minimize the capillary end-effect than the traditional bump flow test, mainly making the saturation profile more uniform over the length of the sample, so in the multiple flow tests it is possible to account for the edge effect.

3. The relative permeability and capillary pressure curves obtained simultaneously in the same experiment reduce the problem of non-uniqueness of the solution, associated with the non-linear regression calculations applied in the historical adjustment.

\section{Acknowledgments}

The authors gratefully acknowledge the support provided by CENPES/PETROBRAS, LRAP/COPPE/UFRJ and CAPES.

\section{References}

1. Cruz DA (2015) Estudo Das Curvas De Permeabilidade Relativa Água-Óleo Considerando O Efeito De Forças
Capilares Na Produção Adicional De Óleo. Programa de Pós-graduação em Engenharia Civil, da Universidade Federal do Rio de Janeiro, Rio de Janeiro, Brazil.

2. Al-Mjeni R, Van Wunnik SAPC, Edwards J, Felber BJ, Gurpinar 0, et al. (2010) Has The Time Come For EOR? Oilfield Review Winter 22(4): 16-35.

3. Johnson EF, Bossler DP, Naumann Bossler VO (1959) Calculation of Relative Permeability from Displacement Experiments. Trans 216(01): 370-372.

4. Gupta R, Maloney DR (2016) Intercept Method--A Novel Technique To Correct Steady-State Relative Permeability Data for Capillary End Effects. SPE Reservoir Evaluation \& Engineering 19(2): 316-330.

5. Handley GF, Handy LL (1956) A Theoretical and Experimental Study of the Steady State Capillary End Effect. Fall Meeting of the Petroleum Branch of AIME, Los Angeles, California, USA.

6. Ucan S, Civan F, Evans R (1997) Uniqueness and Simultaneous Predictability of Relative Permeability and Capillary Pressure by Discrete and Continuous Means. J Can Pet Technol 36(4): 52-61.

7. Lenormand R, Lenormand G (2016) Recommended Procedure For Determination Of Relative Permeabilities. International Symposium of the Society Core Analysis held in Snowmassa, Colorado, USA, pp: 1-12.

8. Rios E Hours, Ramos PFO, Compan ALM, Surmas R, Camargo Hours VR, et al. (2012) Laboratory petrophysics in support of exploration and characterization of reservoirs. Brazilian Symposium on Geophysics.

9. Ambrus J, Maliska C, Hurtado FSV, Silva AFC (2004) Uma metodologia de estimação de parâmetros aplicada à determinação de curvas de permeabilidade relativa de rochas reservatório. Iberian Latin-American Congress 


\section{Petroleum \& Petrochemical Engineering Journal}

on Computational Methods in Engineering, Brazil.

10. Bauget F, Gautier S, Lenormand R, Samouillet A (2012) Gas-Liquid Relative Permeabilities From One-Step And Multi-Step Centrifuge Experiments. International Symposium of the Society of Core Analysts held in Aberdeen, Scotland, UK, pp: 1-12.

11. Jones SC, Roszelle WO (1978) Graphical Techniques for Determining Relative Permeability From Displacement Experiments. J Pet Technol 30(5): 807-817.

12. Viegas VT (2017) Comparação Entre Os Regimes Transiente E Permanente Na Obtenção De Curvas De Permeabilidade Relativa. Programa de Pós-graduação em Engenharia Civil, COPPE, da Universidade Federal do Rio de Janeiro, Brazil.

13. Rosa AJ, Carvalho RS, Xavier JAD (2006) Engenharia de Reservatórios de Petróleo. Editora Interciência Ltda, Rio de Janeiro, Brasil.

14. Wang S (2014) Measurement of Relative Permeabilities at Low Saturation using a Multi-step Drainage Process.
University of Calgary, Alberta, Canada.

15. Lomeland F, Ebeltoft E, Hammervold Thomas W (2005) A New Versatile Relative Permeability Correlation. International Symposium of the SCA, Canada, pp: 1-12.

16. Cydarex, User Manuals, Common Features.

17. Abdallah W, Graue A, Fordham EJ, Habashy T (2007) Fundamentals of wettability. Oilfield Review 19(2): 4461.

18. Masalmeh SK (2012) Impact of capillary forces on residual oil saturation and flooding experiments for mixed to oil-wet carbonate reservoirs. International Symposium of the Society of Core Analysts held in Aberdeen, Scotland, UK, pp: 1-14,27.

19. Crotti MA (2008) Motion of Fluids. Oil \& Gas Reservoirs, InLab.

20. Peters EJ (2012) Advanced Petrophysics: Dispersion, Interfacial Phenomena Wettability, Capillarity Capillary Pressure, Relative Permeability. Vol 2, Live Oak Book Company, pp: 276. 